OPEN ACCESS

Edited by:

Jinhu Tian,

Zhejiang University, China

Reviewed by:

Demin Cai,

Yangzhou University, China

Zhongkai Zhou,

Tianjin University of Science and

Technology, China

Yan Zhang,

China Agricultural University, China Haibo Pan

Zhejiang University, China

${ }^{*}$ Correspondence:

Junjun Wang

wangjj@cau.edu.cn

Specialty section: This article was submitted to Nutrition and Microbes, a section of the journal

Frontiers in Nutrition

Received: 03 June 2021 Accepted: 09 December 2021 Published: 10 January 2022

Citation:

Bai Y, Zhou X, Zhao J, Wang Z, Ye H, Pi Y, Che D, Han D, Zhang S and Wang J (2022) Sources of Dietary Fiber Affect the SCFA Production and Absorption in the Hindgut of Growing

Pigs. Front. Nutr. 8:719935. doi: 10.3389/fnut.2021.719935

\section{Sources of Dietary Fiber Affect the SCFA Production and Absorption in the Hindgut of Growing Pigs}

\author{
Yu Bai ${ }^{1}$, Xingjian Zhou ${ }^{1}$, Jinbiao Zhao ${ }^{1}$, Zhenyu Wang ${ }^{1}$, Hao Ye ${ }^{1}$, Yu Pi ${ }^{1,2}$, \\ Dongsheng Che ${ }^{1,3}$, Dandan Han ${ }^{1}$, Shuai Zhang ${ }^{1}$ and Junjun Wang ${ }^{1 *}$ \\ ${ }^{1}$ State Key Laboratory of Animal Nutrition, College of Animal Science and Technology, China Agricultural University, Beijing, \\ China, ${ }^{2}$ State Key Laboratory of Biological Feed, Ministry of Agriculture and Rural Affairs, Boen Biotechnology Co. Ltd., \\ Ganzhou, China, ${ }^{3}$ College of Animal Science and Technology, Jilin Agricultural University, Changchun, China
}

Effects of different dietary fiber (DF) sources on short-chain fatty acids (SCFA) production and absorption in the hindgut of growing pigs were studied by an in vivo-vitro (ileal cannulated pigs and fecal inoculum-based fermentation) method. Thirty-six cannulated pigs (body weight: $48.5 \pm 2.1 \mathrm{~kg}$ ) were randomly allocated to 6 treatments containing the same DF content (16.5\%), with either wheat bran (WB), corn bran (CB), sugar beet pulp (SBP), oat bran (OB), soybean hulls $(\mathrm{SH})$, or rice bran $(\mathrm{RB})$ as $\mathrm{DF}$ sources. Pigs were allowed 15 days for diet adaptation, and then, fresh ileal digesta and feces were collected to determine SCFA concentration which was normalized for food dry matter intake (DMI) and the hindgut DF fermentability. Fecal microbiota was inoculated into the freeze-dried ileal digesta samples to predict the ability of SCFA production and absorption in the hindgut by in vitro fermentation. The SH group had the largest concentration of total SCFA and propionate in ileal digesta and fecal samples of growing pigs ( $p<0.05)$. Nonetheless, the predicted acetate, total SCFA production, absorption in the SBP group were the highest $(p<0.01)$, but the lowest in the OB group $(p<0.01)$ among all groups. Even SBP and OB group had a similar ratio of soluble DF (SDF) to insoluble DF (IDF). The CB group had high determined ileal and fecal butyrate concentration but the lowest butyrate production and absorption in the hindgut $(p<0.01)$. Overall, the source of DF had a great impact on the hindgut SCFA production and absorption, and SBP fiber had a great potential to increase hindgut SCFA production and absorption.

Keywords: fiber sources, in vivo-vitro method, SCFA production and absorption, hindgut fermentation, growing pigs

\section{INTRODUCTION}

As one of the major dietary components, dietary fiber (DF) could hardly be digested in the foregut due to the lack of corresponding enzymes but could be fermented by microbiota in the gastrointestinal tract (GIT) (1). Short-chain fatty acids are the main metabolites of DF fermentation, including acetate, propionate, and butyrate. Many reports indicated that SCFA was helpful to prevent intestinal diseases, promote proliferation of GIT tissue and the absorption of minerals, and reduce the level of cholesterol in the blood $(2,3)$. Besides, SCFA produced in the hindgut was an important energy source for humans and pigs. It had been reported that $10-13 \%$ of the energy requirement in humans and pigs were supplied by SCFA $(4,5)$. 
Wheat bran (WB), corn bran (CB), sugar beet pulp (SBP), oat bran $(\mathrm{OB})$, soybean hulls $(\mathrm{SH})$, and rice bran $(\mathrm{RB})$ have been widely used in foods and feeds as ubiquitous fibrous ingredients. $\mathrm{DF}$ is the core component of these fibrous ingredients, and the ability of DF to produce SCFA in the GIT is the essential fermentation characteristic of these fibrous ingredients. Fermentation characteristic of DF in fibrous ingredients has been explored in many trials. But these researches had just concluded that the fermentation of $\mathrm{CB}, \mathrm{SB}$, and $\mathrm{WB}$ had a preference for butyrate production in weaned pig's hindgut, or WB was easier to be fermented into SCFA than OB $(6,7)$. However, the SCFA concentration in these researches could not reveal the true SCFA production ability of DF, because DF in commercial diets was not only origin from fibrous ingredients but also the other feedstuffs, such as corn, soybean meal, and so on.

Furthermore, the SCFA in feces was assumed as the ability of SCFA production in the hindgut in previous human and animal research $(8,9)$. Short-chain fatty acids existed in digesta and feces could not represent the true production ability of SCFA, but the remnant of SCFA absorbed in the hindgut. Short-chain fatty acids in the hindgut were absorbed by epithelium and diffused into the portal vein, and the SCFA excreted in the feces only accounted for $5 \%$ of SCFA production in the hindgut $(10,11)$. Besides, short-chain fatty acids consumed by bacteria cannot be neglected, because bacteria were known to cross-feeding on SCFA (12). An in vivo-vitro method was used to estimate the amount of SCFA production and absorption in the hindgut (13). Ileal digesta was served as a substrate and inoculated with fecal microbiota to predict the SCFA production in the hindgut (simulate the fermentation activity in the hindgut). Finally, short-chain fatty acids in fresh ileal digesta, fecal sample and production in vitro were applied to calculate the amount and proportion of SCFA absorbed in the hindgut (14). Notwithstanding, the effect of DF solubility on the SCFA production and absorption in the hindgut had been investigated in the previous research (15), but the interference of other sources of DF and the interactions between the different sources of DF were neglected.

This study used the growing pig model to predict the impact of different sources of DF on SCFA production and absorption in the hindgut by an in vivo-vitro method.

\section{MATERIALS AND METHODS}

This study followed the Laboratory Animal Welfare and Animal Experimental Ethical Inspection Committee in China Agricultural University (AW32110202-2). The in vivo trial was conducted at the Swine Research Unit of China Agricultural University (Beijing, China).

\section{Experimental Diets and Pigs}

$\mathrm{WB}, \mathrm{CB}, \mathrm{SBP}, \mathrm{OB}, \mathrm{SH}$, and $\mathrm{RB}$ were selected as the only DF source for each treatment. The total dietary fiber (TDF) in the diets was contained the same level (16.5\%) through modulating the inclusion level of fibrous ingredients. The result of diet chemical compositions and the feed ingredients were shown in Table 1. All diets were formulated to provide enough or exceed vitamins and minerals for experimental animals based on nutrient requirements of swine (16). $0.3 \%$ chromic oxide was added as indigestible marker for the determination of digestibility. Thirtysix (Duroc $\times$ Landrace $\times$ Large White) crossbred barrows (initial body weight of $48.5 \pm 2.1 \mathrm{~kg}$ ) were operated with a T-cannula in the distal ileum, $\sim 5 \mathrm{~cm}$ cranial to the ileocecal sphincter. All pigs were housed in individual stainless-steel metabolism crates $(1.3 \times 0.8 \times 0.7 \mathrm{~m})$ and allowed 14 days for recovery after surgery. Surgical and nursing procedures were following the Stein's research (17).

\section{Experimental Design and Sample Collection in vivo Trial}

A total of 36 pigs were randomly allocated to the six dietary treatments (six pigs/diet) and provided water ad libitum. The estimated requirement for maintenance energy (i.e., $197 \mathrm{Kcal}$ $\mathrm{ME} / \mathrm{kg}$ body weight ${ }^{0.6}$ ) was provided with pigs for 3 times (16). Two equivalent daily meals were provided at 08:30 and 16:30. The animal experiment lasted for 21 days, including the first 15 days for diet adaptation, and all feces were collected on the 16th, 17 th, and 18th day. Ileal digesta was collected on the last 3 days (19th, 20th, and 21th day) and collected for 16 hours per day until no digesta outflows. A total of 36 samples of ileal digesta were collected for in vitro fermentation assay. Fresh feces were collected by the rectal palpation technique and stored at $-80^{\circ} \mathrm{C}$ for fecal SCFA determination. Feces naturally excreted by pigs were collected in sterile plastic bags immediately and saved in $-20^{\circ} \mathrm{C}$ condition. For ileal digesta collection, sterile polyethylene bags were used to collect ileal digesta by attaching barrel of the cannula. Bags were replaced every 25 min unless filled with ileal digesta and stored at $-20^{\circ} \mathrm{C}$. The first bag of fresh digesta was sampled and stored at $-80^{\circ} \mathrm{C}$ immediately for ileal SCFA determination. At the end of the animal trail, all samples were pooled based on pig and collection date. Ileal digesta and feces were freeze-dried, mixed sufficiently, and stored at $-20^{\circ} \mathrm{C}$.

\section{In vitro Fermentation Assay}

The procedure of ileal digesta fermentation and fecal inoculum preparation was based on the previous studies $(14,18)$. In brief, fresh feces were collected from six growing pigs $[50 \mathrm{~kg}$ bodyweight approximately and fed with commercial grower diet (Supplementary Table 1)] without the use of antibiotics in the last 3 months before feces collection. Then, the feces were homogenized with sterile $0.1 \mathrm{M}$ PBS at $\mathrm{pH} 7(1: 5, \mathrm{w} / \mathrm{v})$ and filtered with four layers of sterile gauzes to serve as the inoculum. Then, $500 \mathrm{mg}$ of freeze-dried ileal digesta (DMI) samples collected in vivo trail was accurately weighed into the serum bottle and added with $25 \mathrm{~mL}$ inoculum. The empty bottles were incubated with $25 \mathrm{~mL}$ of phosphate buffer and served as blank control. The serum bottles were flushed with $\mathrm{CO}_{2}$ and capped immediately after inoculation and then transported to a prewarmed $\left(37^{\circ} \mathrm{C}\right)$ incubator for $38 \mathrm{~h}$. All the procedures were conducted in a condition filled with $\mathrm{CO}_{2}$ at $37^{\circ} \mathrm{C}$. Four replicate bottles were prepared for substrate or blank, one for SCFA determination and the other for the determination of dry matter (DM) fermentability. After incubation, the fermentation broth was centrifuged at $15,000 \mathrm{~g}$ for $12 \mathrm{~min}$ at $4^{\circ} \mathrm{C}$. The fermentation broth supernatant was collected after centrifugation and stored 
TABLE 1 | Ingredients and nutrient compositions of the diets.

\begin{tabular}{|c|c|c|c|c|c|c|}
\hline & \multicolumn{6}{|c|}{ Diets $^{a}$} \\
\hline Corn starch & 35.08 & 46.36 & 45.95 & 34.63 & 45.13 & 21.43 \\
\hline Soy protein isolate & 12.00 & 12.00 & 14.00 & 12.00 & 14.00 & 11.00 \\
\hline WB & 35.70 & - & - & - & - & - \\
\hline SBP & - & - & 22.50 & - & - & - \\
\hline OB & - & - & - & 35.80 & - & - \\
\hline $\mathrm{SH}$ & - & - & - & - & 23.50 & - \\
\hline RB & - & - & - & - & - & 50.00 \\
\hline Soy oil & 30.00 & 30.00 & 30.00 & 30.00 & 30.00 & 30.00 \\
\hline $\mathrm{NaCl}$ & 0.45 & 0.45 & 0.45 & 0.45 & 0.45 & 0.45 \\
\hline $\mathrm{K}_{2} \mathrm{CO}_{3}$ & 0.30 & 0.30 & 0.30 & 0.30 & 0.30 & 0.30 \\
\hline $\mathrm{MgO}$ & 0.10 & 0.10 & 0.10 & 0.10 & 0.10 & 0.10 \\
\hline L-lysine-HCl & 0.40 & 0.48 & 0.35 & 0.42 & 0.30 & 0.32 \\
\hline DL-Methionine & 0.12 & 0.16 & 0.15 & - & 0.12 & 0.12 \\
\hline L-Threonine & 0.15 & 0.20 & 0.20 & 0.20 & 0.20 & 0.18 \\
\hline Premix ${ }^{b}$ & 0.50 & 0.50 & 0.50 & 0.50 & 0.50 & 0.50 \\
\hline \multicolumn{7}{|l|}{ Nutrient (\%, DM) } \\
\hline GE (MJ/kg DM) & 1.82 & 1.85 & 1.82 & 1.80 & 1.83 & 1.81 \\
\hline $\mathrm{CP}$ & 12.80 & 13.32 & 13.76 & 14.28 & 13.33 & 15.22 \\
\hline Cellulose & 3.06 & 3.92 & 4.32 & 2.00 & 9.29 & 1.03 \\
\hline Hemicellulose & 10.20 & 13.29 & 7.64 & 14.81 & 7.31 & 9.09 \\
\hline
\end{tabular}

aWB, wheat bran; $C B$, corn bran; SBP, sugar beet pulp; OB, oat bran; $S H$, soybean hulls; RB, rice bran.

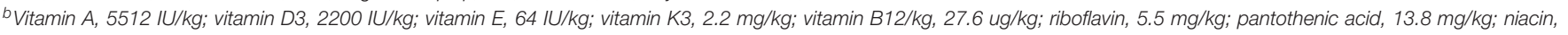

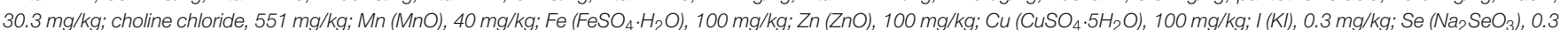

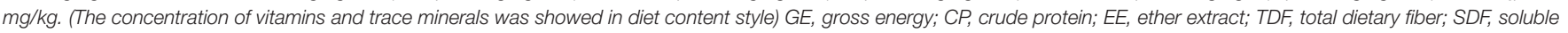
dietary fiber; IDF, insoluble dietary fiber; NDF, neutral detergent fiber; ADF, acid detergent fiber.

at $-80^{\circ} \mathrm{C}$ until analyzed for SCFA concentration. The DM of the unfermented residue was determined by drying them at $65^{\circ} \mathrm{C}$ until constant weight reached after sterilization.

\section{Chemical Analyses}

The diets, ileal digesta, and feces were analyzed in duplicate for DM, chromium, SDF, IDF, neutral detergent fiber (NDF), acid detergent fiber $(\mathrm{ADF})$, acid detergent lignin $(\mathrm{ADL})$, cellulose, and hemicellulose. The diets were also determined for gross energy (GE), ether extract (EE), and cross-protein (CP). The DM (Method 934.01), EE (Method 920.39), crude protein (CP)
(Method 990.03), chromium (Method 990.08), soluble DF (SDF), and insoluble DF (IDF) (Method 991.43) in the diets were also analyzed (19). NDF and ADF were determined by the method of Van Soest et al. (20). Hemicellulose was seen as the difference between NDF and ADF, and cellulose was the difference between $\mathrm{ADF}$ and ADL.

The concentration of SCFA in ileal digesta, fecal samples, and fermentation supernatants was determined by previous method (21). The sum of the acetate, propionate, and butyrate was seen as total SCFA, and the branch-chain fatty acids were not considered in this research. 


\section{Calculation and Statistical Analysis In vivo Assay}

The determined hindgut fermentability was calculated as follows:

$$
\begin{aligned}
& \text { Determined hindgut fermentability } \text { in vivo }(\%) \\
& \quad=\left(1-\left(\left(\mathrm{DM}_{\mathrm{F}} \div \mathrm{DM}_{\mathrm{I}}\right) \times\left(\mathrm{T}_{\mathrm{I}} \div \mathrm{T}_{\mathrm{F}}\right)\right)\right) \times 100
\end{aligned}
$$

where $T_{I}$ and $T_{F}$ are the chromic oxide contents $(\mathrm{g} / \mathrm{kg} \mathrm{DM})$ in ileal digesta and fecal samples; $\mathrm{DM}_{\mathrm{F}}$ and $\mathrm{DM}_{\mathrm{I}}$ are the contents of $\mathrm{DM}(\mathrm{g} / \mathrm{kg})$ in fecal samples or ileal digesta, respectively.

The concentration of SCFA in ileal digesta and fecal samples (normalized for DMI) was calculated according to the research of Montoya et al. (14).

$$
\begin{aligned}
& \text { Normalized SCFA concentration }(\mathrm{mmol} / \mathrm{kg} \mathrm{DMI}) \\
& \quad=\text { SCFA concentration }(\mathrm{mmol} / \mathrm{kg} \mathrm{DM}) \times\left(\mathrm{T}_{\mathrm{D}} \div \mathrm{T}_{\mathrm{F} / \mathrm{I}}\right)
\end{aligned}
$$

where $\mathrm{T}_{\mathrm{D}}, \mathrm{T}_{\mathrm{F}}$, and $\mathrm{T}_{\mathrm{I}}$ are the chromic oxide content (g/kg DM) in the diet, fecal samples, and ileal digesta, respectively.

\section{In vitro Fermentation Assay}

The fermentability of DM and the production of SCFA were seen as the predicted hindgut fermentability of DM, and the production of SCFA in the hindgut was calculated as follows:

$$
\begin{aligned}
& \text { Predicted hindgut fermentability } \text { in vitro }(\%) \\
& =\left(\mathrm{DM}_{\mathrm{b}}-\left(\mathrm{DM}_{\mathrm{a}}-\left(\mathrm{DM}_{\text {blank initial }}+\mathrm{DM}_{\text {blank final }}\right) / 2\right)\right) / \\
& \quad D M_{b} \times 100 \\
& \text { Production of SCFA during fermentation } \\
& \quad\left(\mathrm{mmol}_{\mathrm{k} g} \mathrm{DM} \text { incubated }\right) \\
& =\left(\mathrm{SCFA}_{\text {sample }}-\left(\frac{\left(\mathrm{SCFA}_{\text {blank initial }}+\mathrm{SCFA}_{\text {blank final }}\right)}{2}\right)\right) / \\
& \quad \text { sample weight }(\mathrm{g} \mathrm{DM}) \times 1,000
\end{aligned}
$$

where $\mathrm{DM}_{\mathrm{b}}$ and $\mathrm{DM}_{\mathrm{a}}$ are the $\mathrm{DM}(\mathrm{mg})$ of the ileal digesta either before or after fermentation. $\mathrm{DM}_{\text {blankinitial }}, \mathrm{DM}_{\text {blankfinal }}$, SCFA blankinitial, and SCFA blankfinal are the DM (mg) and the SCFA (mmol) in the blank bottle before (initial) and after (final) fermentation, respectively (22).

The predicted SCFA production was calculated based on the in vivo-vitro combining result (ileal cannulated pig and mimic hindgut fermentation). The predicted hindgut SCFA production was calculated as following equations (14):

$$
\begin{aligned}
& \text { Predicted hindgut SCFA production }(\mathrm{mmol} / \mathrm{kg} \mathrm{DMI}) \\
& =\text { SCFA produced by fermentation } \\
& \quad(\mathrm{mmol} / \mathrm{kg} \text { ileal digesta DM incubated }) \\
& \quad \times \text { Ileal DM flow }(\mathrm{kg} \mathrm{DM} / \mathrm{kg} \mathrm{DMI})
\end{aligned}
$$

where ileal DM flow (g/kg DMI) is the ileal flow of DM.

Short-chain fatty acids absorption were predicted by the combined results of SCFA entering and producing in the hindgut (14):

Amounts of SCFA absorbed in the hindgut (mmol/kg DMI)

$=$ in vitro predicted hindgut SCFA production ( $\mathrm{mmol} /$

$\mathrm{kg}$ DMI) + ileal SCFA concentration ( $\mathrm{mmol} / \mathrm{kg} \mathrm{DMI})$

- fecal SCFA concentration ( $\mathrm{mmol} / \mathrm{kg} \mathrm{DMI})$

The extent of SCFA absorbed in the hindgut (\%)

$=$ amount of SCFA absorbed in the hindgut ( $\mathrm{mmol} /$

$\mathrm{kg} \mathrm{DM}$ intake)/(in vitro predicted hindgut SCFA production

$(\mathrm{mmol} / \mathrm{kg} \mathrm{DMI})+$ ileal SCFA concentration

(mmol/kg DMI))

\section{Statistical Analysis}

Statistical analysis was conducted by SPSS software package (SPSS v. 20.0, SPSS Inc, Chicago, IL, USA). Kruskal-Wallis test was performed to test the impact of different fiber sources on the data of in vivo trail and in vitro fermentation trail. The ileal and fecal data were compared by Mann-Whitney test. $p<0.05$ was considered significant.

\section{RESULTS}

\section{SCFA Concentration in lleal Digesta and Feces}

Pigs fed diets containing $\mathrm{CB}$ and $\mathrm{SH}$ had the highest $(p<0.01)$ normalized ileal acetate among all groups (Table 2), and the $\mathrm{SH}$ group also had the highest $(p<0.01)$ normalized ileal total SCFA, propionate, and butyrate. In contrast to the $\mathrm{SH}$ group, the normalized ileal total SCFA, acetate, and propionate in the OB group were the lowest $(p<0.01)$ among all groups. The WB group had the lowest $(p<0.01)$ normalized ileal butyrate as well. The normalized total SCFA and propionate in the fecal samples of SH group were higher $(p<0.05)$ than that of SBP, $\mathrm{WB}, \mathrm{OB}$, and $\mathrm{RB}$. The normalized acetate concentration in fecal samples was higher $(p<0.05)$ than that in ileal digesta for all groups. Furthermore, the determined ileal and fecal SCFA in the SH treatment were the highest $(p<0.05)$ among all groups except ileal acetate (Supplementary Table 2). However, the RB group showed the lowest $(p<0.05)$ determined fecal SCFA concentration.

\section{Short-Chain Fatty Acids Production During in vitro Fermentation and Predicted SCFA Production in the Hindgut}

The production of total SCFA, acetate, and propionate in vitro was the highest $(p<0.01)$ in SBP group but the lowest $(p$ $<0.01$ ) in OB group, and also predicted total SCFA, acetate, and propionate production $(p<0.01)$ in the hindgut (Table 3 ). Besides, the propionate and butyrate production in vitro and predicted production in the hindgut of the $\mathrm{CB}$ group were the lowest $(p<0.01)$ among all groups. Butyrate production in WB group was the highest $(p<0.01)$, but there was no difference in predicted production of butyrate in hindgut among the WB, SBP, $\mathrm{OB}, \mathrm{SH}$, and $\mathrm{RB}$ groups. 
TABLE 2 | Normalized ileal and fecal SCFA concentration for ileal cannulated pigs fed diets containing different sources of fiber ( $n=6$ per group).

\begin{tabular}{|c|c|c|c|c|c|c|c|c|}
\hline \multirow[b]{2}{*}{ Items } & \multicolumn{6}{|c|}{ Diets $^{1}$} & \multirow[b]{2}{*}{ SEM } & \multirow[b]{2}{*}{$P$-value } \\
\hline & WB & CB & SBP & OB & SH & RB & & \\
\hline \multicolumn{9}{|c|}{ Normalized ileal SCFA (mmol/kg DMI) (in vivo) } \\
\hline Acetate & $150.70^{a b}$ & $263.62^{a}$ & $256.71^{a b}$ & $125.74^{b}$ & $275.68^{a}$ & $247.88^{a b}$ & 23.02 & $<0.01$ \\
\hline Propionate & $41.51^{b}$ & $44.83^{b}$ & $15.62^{b}$ & $3.84^{b}$ & $129.66^{a}$ & $27.63^{\mathrm{b}}$ & 8.58 & $<0.01$ \\
\hline Butyrate & $2.27^{c}$ & $8.15^{\mathrm{abc}}$ & $7.09^{b c}$ & $15.26^{\mathrm{ab}}$ & $17.60^{\mathrm{a}}$ & $7.01^{b c}$ & 1.74 & $<0.01$ \\
\hline Total SCFA ${ }^{2}$ & $194.48^{b c}$ & $316.59^{a b}$ & $279.42^{\mathrm{abc}}$ & $144.85^{c}$ & $422.94^{a}$ & $282.52^{\mathrm{abc}}$ & 25.33 & $<0.01$ \\
\hline \multicolumn{9}{|c|}{ Normalized fecal SCFA (mmol/kg DMI) (in vivo) } \\
\hline Acetate & 94.16 & 119.05 & 86.67 & 74.62 & 114.23 & 97.12 & 10.08 & $>0.05$ \\
\hline Propionate & $38.16^{\mathrm{b}}$ & $59.90^{\mathrm{ab}}$ & $31.20^{\mathrm{b}}$ & $29.02^{b}$ & $78.82^{\mathrm{a}}$ & $35.53^{\mathrm{b}}$ & 6.97 & $<0.01$ \\
\hline Butyrate & 21.93 & 26.77 & 21.76 & 23.87 & 42.20 & 11.39 & 6.18 & $>0.05$ \\
\hline Total SCFA & $154.25^{\mathrm{b}}$ & $205.72^{a b}$ & $139.63^{b}$ & $127.52^{b}$ & $235.25^{\mathrm{a}}$ & $144.04^{b}$ & 22.20 & $<0.05$ \\
\hline \multicolumn{9}{|c|}{ Statistical analysis for ileal vs. fecal SCFA concentration ${ }^{3}$} \\
\hline$P_{\text {Acetate }}$ & $<0.05$ & $<0.05$ & $<0.05$ & $<0.05$ & $<0.01$ & $<0.01$ & & \\
\hline SEM & 18.89 & 24.78 & 26.44 & 11.33 & 10.41 & 7.43 & & \\
\hline P Propionate & $>0.05$ & $>0.05$ & $<0.05$ & $<0.01$ & $<0.05$ & $>0.05$ & & \\
\hline SEM & 15.06 & 15.01 & 2.14 & 3.72 & 6.49 & 4.21 & & \\
\hline$P_{\text {Butyrate }}$ & $<0.05$ & $<0.01$ & $<0.05$ & $<0.05$ & $<0.05$ & $<0.01$ & & \\
\hline SEM & 3.94 & 2.72 & 4.21 & 4.25 & 6.29 & 2.35 & & \\
\hline$P_{\text {Total SCFA }}$ & $>0.05$ & $<0.05$ & $<0.05$ & $>0.05$ & $<0.05$ & $<0.01$ & & \\
\hline SEM & 29.95 & 35.11 & 31.99 & 17.34 & 21.86 & 6.33 & & \\
\hline
\end{tabular}

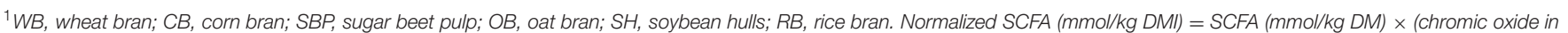
the diets $\div$ chromic oxide content in feces or ileal digesta).

${ }^{2}$ Total SCFA $=$ acetate + propionate + butyrate.

${ }^{3} P_{\text {Acetate }}, P_{\text {Propionate }} P_{\text {Butyrate, }}$ and $P_{\text {Total SCFA }}$ are the $p$-values for comparing ileal and fecal acetate, propionate, butyrate, and total SCFA, respectively.

$a, b, c$ Values in the same row with different letter superscripts means significant difference $(p<0.05)$.

\section{Predicted SCFA Absorption in the Hindgut}

The predicted total SCFA, acetate, propionate, and butyrate absorption in the hindgut were the highest $(p<0.01)$ in SBP group (Table 3). The lowest $(p<0.01)$ amount of acetate but the highest $(p<0.01)$ amount of butyrate absorption was found in the hindgut of pigs fed OB-containing diet. The lowest $(p<0.01)$ absorption of butyrate and propionate also occurred in CB group.

Pigs in the SBP group had the higher $(p<0.01)$ predicted apparent total SCFA, acetate, and propionate absorption in the hindgut than SH group (Table 3). The predicted apparent absorption of total SCFA, propionate, and butyrate in SBP group was also higher $(p<0.01)$ than that of CB group. In addition, the predicted apparent absorption of propionate and butyrate in $\mathrm{CB}$ group was the lowest $(p<0.01)$.

\section{Hindgut Fermentability of Fiber for Growing Pigs Fed Diets Containing the Different Sources of Fiber}

The SBP, OB, and SH group had the higher $(p<0.01)$ hindgut $\mathrm{NDF}$ fermentability than $\mathrm{WB}, \mathrm{CB}$, and $\mathrm{RB}$ group, and there was no difference in the hindgut fermentability of NDF among the SBP, OB, and SH groups (Table 4). Besides, the hindgut fermentability of SDF and IDF had the same trend as that of NDF among all groups. The hindgut fermentability of ADF in $\mathrm{WB}$ and $\mathrm{RB}$ group was the lowest $(p<0.01)$. The $\mathrm{CB}$ and $\mathrm{RB}$ group had the lower $(p<0.01)$ hindgut fermentability of cellulose and hemicellulose than SBP and OB group.

\section{Determined and Predicted Hindgut DM Fermentability for Growing Pigs Fed Diets Containing Different Sources of Fiber}

The SBP, OB, and $\mathrm{SH}$ group were found to have the higher $(p<0.01)$ hindgut DM fermentability than $\mathrm{WB}, \mathrm{CB}$, and $\mathrm{RB}$, and there was no difference in the hindgut fermentability of $\mathrm{DM}$ among the SBP, OB, and SH group (Table 5). Furthermore, the SBP group had the highest $(p<0.01)$ predicted hindgut DM fermentability followed by the OB group, and the predicted hindgut DM fermentability for WB and CB groups was the lowest $(p<0.01)$. The predicted hindgut DM fermentability was higher $(p<0.05)$ than the determined hindgut DM fermentability in $\mathrm{OB}$ group, whereas there were no differences in the other groups.

\section{DISCUSSION}

This research investigated the impact of different DF sources on the predicted SCFA production and absorption in the hindgut using pig model. Recently, the probiotic characteristics of DF have attracted huge amounts of interest, and WB, CB, SBP, OB, $\mathrm{SH}$, and $\mathrm{RB}$ are ubiquitous fibrous ingredients which had been 
TABLE 3 | Predicted SCFA production and absorption in hindgut (in vivo-vitro assay) of pigs fed the diets with different sources of fiber ( $n=6$ per group).

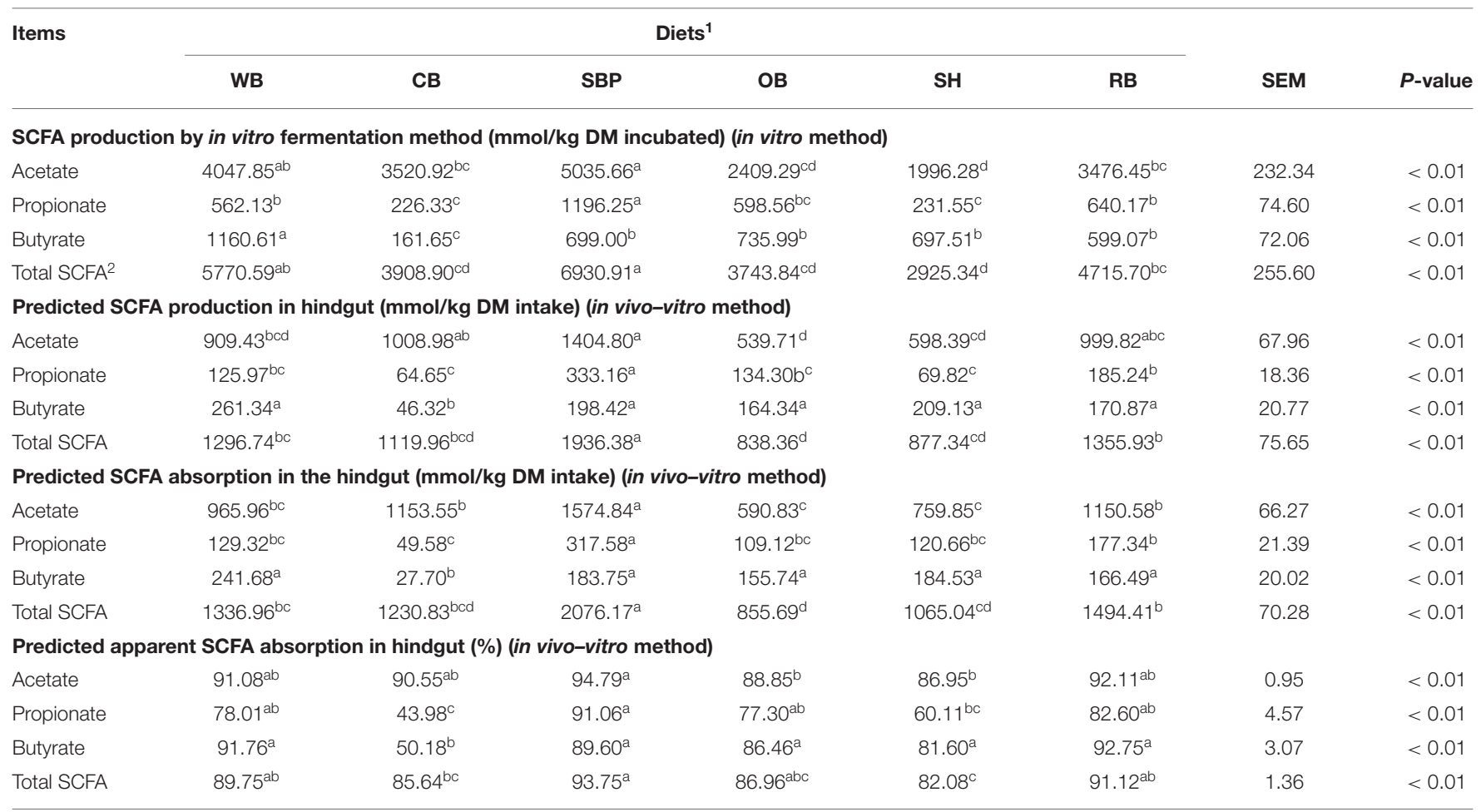

${ }^{1}$ WB, wheat bran; $C B$, corn bran; SBP, sugar beet pulp; $O B$, oat bran; SH, soybean hulls; RB, rice bran.

${ }^{2}$ Total SCFA $=$ acetate + propionate + butyrate.

${ }^{a-d}$ Values in the same row with different letter superscripts means significant difference $(p<0.05)$.

TABLE 4 | Determined apparent hindgut fiber fermentability for ileal cannulated pigs fed the diets with different sources of fiber ( $n=6$ per group).

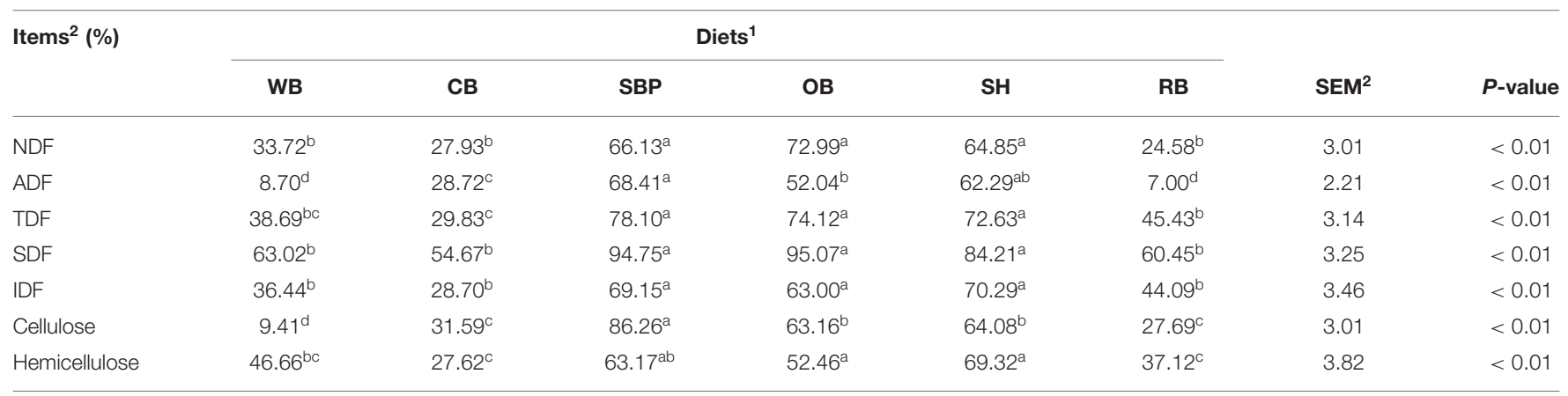

${ }^{1}$ WB, wheat bran; $C B$, corn bran; $S B P$, sugar beet pulp; $O B$, oat bran; $S H$, soybean hulls; RB, rice bran.

${ }^{2} N D$, neutral detergent fiber; $A D F$, acid detergent fiber; TDF, total dietary fiber; $S D F$, soluble dietary fiber; IDF, insoluble dietary fiber.

${ }^{a-d}$ Values in the same row with different letter superscripts means significant difference $(p<0.05)$.

extensively used in foods and feeds. Fermentation characteristics of DF for these fibrous ingredients in the hindgut are vital parts of the knowledge about the rational use of DF. Some studies tried to elucidate the fermentation characteristic of the DF contained in the fibrous feedstuffs and investigated the SCFA production in GIT by focusing on the fecal SCFA, even the ileal SCFA $(23,24)$. Nonetheless, short-chain fatty acids existing in the feces and ileal digesta could not represent the production of SCFA in GIT
(25). The diet is digested and partially fermented by bacteria into SCFA in the upper tract after ingested by pigs. Part of the SCFA produced in the upper tract is absorbed by the host, and the others flowed into the hindgut accompanied by undigested nutrients. Short-chain fatty acids produced in the hindgut is derived from the ileal digesta fermentation by microbes in the hindgut. The SCFA in the hindgut was mainly absorbed by the host as the energy for the epithelium and enter the blood 
TABLE 5 | Determined and predicted hindgut DM fermentability for ileal cannulated pigs fed the diets with different sources of fiber ( $n=6$ per group).

\begin{tabular}{|c|c|c|c|c|c|c|c|c|}
\hline \multirow[t]{2}{*}{ Items } & \multicolumn{8}{|c|}{ Diets $^{1}$} \\
\hline & WB & CB & SBP & OB & SH & RB & SEM $^{2}$ & $P$-value \\
\hline \multicolumn{9}{|c|}{ Determined and predicted hindgut $\mathrm{DM}^{2}$ fermentability } \\
\hline Determined fermentability, \% (in vivo) & $35.76^{\mathrm{b}}$ & $36.15^{\mathrm{b}}$ & $66.34^{\mathrm{a}}$ & $56.58^{\mathrm{a}}$ & $60.28^{a}$ & $33.60^{b}$ & 3.09 & $<0.01$ \\
\hline Predicted fermentability, \% (in vivo-in vitro) & $35.66^{\mathrm{e}}$ & $31.17^{e}$ & $73.21^{\mathrm{a}}$ & $67.00^{\mathrm{b}}$ & $54.76^{c}$ & $41.03^{d}$ & 0.86 & $<0.01$ \\
\hline \multicolumn{9}{|c|}{ Comparison of determined and predicted DM fermentability } \\
\hline$P_{\text {hindgutfermentability }}^{3}$ & 0.97 & 0.31 & 0.26 & 0.02 & 0.23 & 0.06 & & \\
\hline SEM & 1.47 & 2.18 & 3.22 & 1.77 & 1.82 & 1.39 & & \\
\hline
\end{tabular}

${ }^{1}$ WB, wheat bran; $C B$, corn bran; SBP, sugar beet pulp; OB, oat bran; SH, soybean hulls; RB, rice bran.

${ }^{2}$ DM, Dry matter.

${ }^{3} P_{\text {hindgutfermentability }}$ is the $p$-value for comparing the determined and predicted hindgut DM fermentability.

${ }^{a-d}$ Values in the same row with different letter superscripts means significant difference $(p<0.05)$.

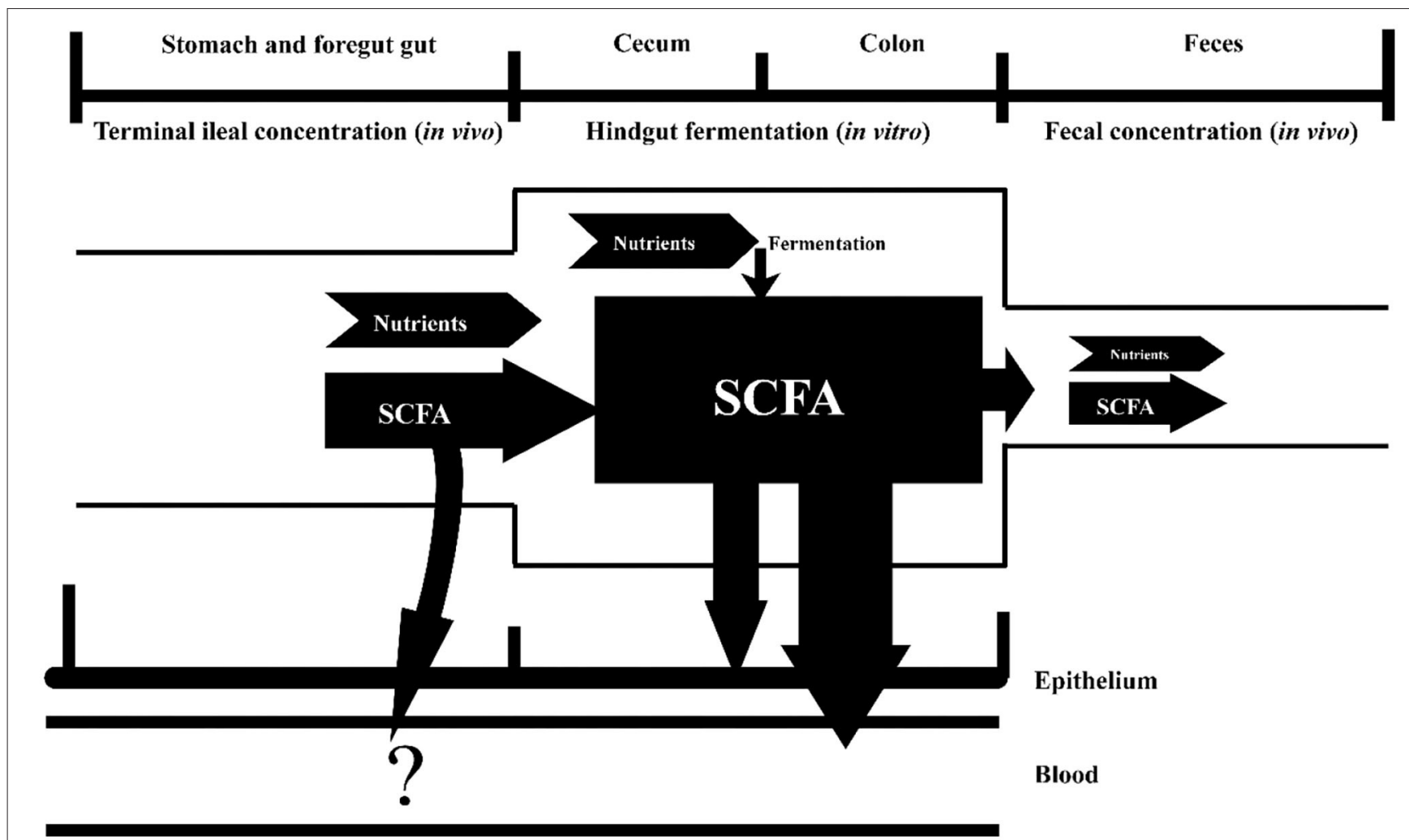

FIGURE 1 | An in vivo-vitro method to determine SCFA production and absorption in the hindgut. The "?" represents the SCFA absorption in the foregut, which was not a measurement in this research.

circulation, and just a small quantity of unabsorbed SCFA and unfermented nutrients were excreted to the outside of the body as feces. The amount of SCFA absorbed in the upper GIT was not accounted in this research (Figure 1).

In this research, an in vivo-vitro method was used to predict the SCFA production and absorption in the hindgut of growing pigs fed with diets containing $\mathrm{WB}, \mathrm{CB}, \mathrm{SBP}, \mathrm{OB}$, and $\mathrm{SH}$, as the only fiber source, respectively. As the experimental setup, the upper tract digestion was conducted in the pig model, and in vitro fermentation trial was conducted to mimic the hindgut fermentation, where ileal digesta served as the substrate and incubated with pig fecal inoculum. By analyzing the combining result of in vivo-vitro trial, we could predict the SCFA production and absorption in the hindgut (26).

The source and solubility of DF are crucial determinants for the fermentation rate and the production of SCFA in the GIT. The SDF was easier to be utilized by microbiota due to its great hydrolysis capacity (27). The previous study researched the effects of SDF and IDF on predicted SCFA production and absorption in the hindgut. Flaxseed meal and oat hulls were used 
as the source of SDF and IDF, respectively, but this research did not confirm the uniqueness of fiber source in diet and neglected the interactions of different source fibers (15). The source of DF is not only affect the solubility of DF but also the composition of DF, which also affecting factors for SCFA production and absorption in the hindgut (28).

The high concentration of ileal acetate and propionate in SBP and SH groups might because of the high content of SDF in SBP and $\mathrm{SH}$ diets. SDF was fermented faster and more extensively by the microbiota compared with the IDF in the GIT (29). The low ileal SCFA but high content of SDF was also found in the $\mathrm{OB}$ group, which verified our hypothesis that solubility and source of DF were affecting the production of SCFA. The higher normalized concentration of fecal SCFA than ileal SCFA was also found in the research of Ndou et al. (15), but contrast to the research of Coles et al. (18). The reason could be the difference in the experimental model, as both Ndou's research and our research were conducted in a pig model, whereas the study of Coles et al. used human feces as the inoculum.

This research found that the predicted acetate and propionate production were the highest in SBP group but the lowest in $\mathrm{OB}$ group, since the ileal digesta of SBP and $\mathrm{OB}$ groups was fermented into the highest and lowest amount of SCFA by in vitro fermentation method, respectively. The great ability of acetate and propionate production of SBP fermentation has been mentioned in our past research, a significant positive correlation was found between cellulose and acetate (24), and the SBP group also had a high hindgut fermentability of cellulose in this research. Wang's research also found the inclusion of SBP caused high SCFA production (30). The low SCFA production capacity in the $\mathrm{OB}$ group was proved by the previous research where pigs fed with a $27 \%$ WB-based diet excreted more acetate, propionate, and total SCFA in feces compared with pigs fed with a diet containing 36\% OB. Pigs fed OB diets inhibited the proliferation of Succinivibrio and Prevotella, which had been confirmed by a close relationship with fiber degradation and SCFA production (31). The lowest predicted apparent propionate and butyrate absorption in CB group were found in this research as well, and the low fermentability of $\mathrm{CB}$ fiber in the hindgut could be the main reason. Determination of fiber fermentability verified our inference that the $\mathrm{CB}$ group was found the lowest TDF, SDF, IDF, and DM fermentability in the hindgut. Meanwhile, propionate and butyrate production in the $\mathrm{CB}$ group were the lowest among all groups. The predicted total SCFA production in the hindgut in $\mathrm{WB}, \mathrm{CB}, \mathrm{SBP}, \mathrm{OB}, \mathrm{SH}$, and $\mathrm{RB}$ group ranged 838 $1,936(\mathrm{mmol} / \mathrm{kg}$ DMI) in this study, whereas the hindgut SCFA production ability of pigs fed the diets containing wheat flour, WB and OB in Christensen et al. study ranged $369-850(\mathrm{mmol} / \mathrm{kg}$ DMI) (26). Multiple fiber sources and high fiber content in diets could be the reason for the wide range of predicted SCFA production. Additionally, the higher body weight of pigs might also contribute to the variation of the range, as pigs with low body weight was found to have lower DF digestibility than pigs with high body weight, leading to high SCFA production in the hindgut (8).

Changes in predicted hindgut SCFA absorption and absorption were commonly consistent with each other. The high estimate of quantities of SCFA absorption in SBP group could be the result of the high quantity of SCFA production, since the SCFA in the large intestine was rapidly absorbed by the host through the passive diffusion way (32). The range of predicted apparent total SCFA absorption in this research was 89.64-96.86\%, which was consistent with the previous study that colonocytes absorbed $90-95 \%$ of the SCFA produced in the humans' and pigs' hindgut (25).

The high hindgut DF fermentability and high level of SCFA production during fermentation (in vitro) were found in the SBP group. The high hindgut fermentability of SDF, NDF, ADF, and cellulose was found when growing pigs fed with diets containing $\mathrm{CB}, \mathrm{SH}$, and SBP (33). However, pigs in the $\mathrm{OB}$ group had the high hindgut DF fermentability as pigs fed with SBP, but the amount of SCFA production of pigs in OB group was less in this research. The reason might be the low IDF content and high SDF content in the OB diet. Microbes in the foregut had been proved the high DF degradation ability, and SDF could be fermented more rapidly by the gut microbes due to its high capacity for hydration compared to IDF (20). A large amount of SDF was degraded in the foregut, only a small amount of IDF was fermented into SCFA in the hindgut. Moreover, the predicted hindgut fermentability of DM (in vitro) was parallel to the determined hindgut DM fermentability (in vivo), which showed the credibility of in vivo-vitro method (22).

\section{CONCLUSION}

Short-chain fatty acids in ileal digesta and feces cannot represent the ability of SCFA production. The DF sources had a great impact on SCFA production and absorption in the hindgut of growing pigs. The fiber of SBP showed the great ability of SCFA production and absorption with high SCFA concentration in ileal digesta and feces. Oat bran fiber showed a low potential for SCFA production and absorption even with high SCFA concentration and hindgut DF fermentability.

\section{DATA AVAILABILITY STATEMENT}

The original contributions presented in the study are included in the article/Supplementary Material, further inquiries can be directed to the corresponding author.

\section{ETHICS STATEMENT}

The animal study was reviewed and approved by Laboratory Animal Welfare and Animal Experimental Ethical Inspection Committee in China Agricultural University.

\section{AUTHOR CONTRIBUTIONS}

$\mathrm{YB}$ and JW designed the study. YB, XZ, and JZ conducted the vivo and vitro experiments. YB analyzed data, performed the statistical analysis, and wrote the paper. ZW, HY, YP, DH, DC, SZ, and 
JW critically reviewed the manuscript. All authors have read and approved the final manuscript.

\section{FUNDING}

This study was supported by the National Natural Science Foundation of China (31972596, 31630074), the Postdoctoral Innovative Talent Support Program (BX20200365), the Beijing

\section{REFERENCES}

1. Tiwari UP, Singh AK, Jha R. Fermentation characteristics of resistant starch, arabinoxylan, and $\beta$-glucan and their effects on the gut microbial ecology of pigs: A review. Anim Nutr. (2019) 5:217-26. doi: 10.1016/j.aninu.2019.04.003

2. Chassaing B, Miles-Brown J, Pellizzon M, Ulman E, Ricci M, Zhang L, et al. Lack of soluble fiber drives diet-induced adiposity in mice. Am J Physiol. (2015) 309:G528-41. doi: 10.1152/ajpgi.00172.2015

3. Tian M, Chen J, Liu J, Chen F, Guan W, Zhang S. Dietary fiber and microbiota interaction regulates sow metabolism and reproductive performance. Anim Nutr. (2020) 6:397-403. doi: 10.1016/j.aninu.2020.10.001

4. Bianchi F, Dall'Asta M, Rio DD, Mangia A, Musci M, Scazzina F, et al. Development of a headspace solid-phase microextraction gas chromatography-mass spectrometric method for the determination of shortchain fatty acids from intestinal fermentation. Food Chem. (2011) 129:200-5. doi: 10.1016/j.foodchem.2011.04.022

5. Hughes SA, Shewry PR, Li L, Gibson GR, Sanz ML, Rastall RA. In vitro fermentation by human fecal microflora of wheat arabinoxylans. J Agric Food Chem. (2007) 55:4589-95. doi: 10.1021/jf070293g

6. Ratanpaul V, Zhang D, Williams BA, Diffey S, Black JL, Gidley MJ. Wheat bran and oat hulls have dose-dependent effects on ad-libitum feed intake in pigs related to digesta hydration and colonic fermentation. Food Funct. (2019) 10:8298-308. doi: 10.1039/C8FO02496K

7. Zhao J, Liu P, Wu Y, Guo P, Liu L, Ma N, et al. Dietary fiber increases butyrateproducing bacteria and improves the growth performance of weaned piglets. J Agric Food Chem. (2018) 66:7995-8004. doi: 10.1021/acs.jafc.8b02545

8. Zhao J, Bai Y, Tao S, Zhang G, Wang J, Liu L, et al. Fiber-rich foods affected gut bacterial community and short-chain fatty acids production in pig model. J Funct Foods. (2019) 57:266-74. doi: 10.1016/j.jff.2019.04.009

9. Zhao J, Zhang G, Liu L, Wang J, Zhang S. Effects of fibre-degrading enzymes in combination with different fibre sources on ileal and total tract nutrient digestibility and fermentation products in pigs. Arch Anim Nutr. (2020) 74:1309-24. doi: 10.1080/1745039X.2020.1766333

10. Jha R, Fouhse JM, Tiwari UP, Li L, Willing BP. Dietary fiber and intestinal health of monogastric animals. Front Vet Sci. (2019) 6:48. doi: $10.3389 /$ fvets. 2019.00048

11. Roy CC, Kien CL, Bouthillier L, Levy E. Short-chain fatty acids: ready for prime time? Nutr Clin Pract. (2006) 21:351-66. doi: $10.1177 / 0115426506021004351$

12. Hoek M, Merks RMH. Emergence of microbial diversity due to cross-feeding interactions in a spatial model of gut microbial metabolism. BMC Syst Biol. (2017) 11:56. doi: 10.1186/s12918-017-0430-4

13. Montoya CA, De Haas ES, Moughan PJ. Development of an in vivo and in vitro ileal fermentation method in a growing pig model. J Nutr. (2018) 148:298-305. doi: 10.1093/jn/nxx038

14. Montoya CA, Rutherfurd SM, Moughan PJ. Kiwifruit fibre level influences the predicted production and absorption of SCFA in the hindgut of growing pigs using a combined in vivo-in vitro digestion methodology. Br J Nutr. (2016) 115:1317-24. doi: 10.1017/S0007114515002883

15. Ndou SP, Kiarie E, Nyachoti CM. Flaxseed meal and oat hulls supplementation: impact on predicted production and absorption of volatile fatty acids and energy from hindgut fermentation in growing pigs. $J$ Anim Sci. (2019) 97:302-14. doi: 10.1093/jas/sky399

16. NRC. Nutrient Requirements of Swine, 11th rev. ed. Washington, DC: National Academies Press (2012).

17. Stein HH, Shipley CF, Easter RA. Technical note: a technique for inserting a T-cannula into the distal ileum of pregnant
Municipal Natural Science Foundation (S170001), and the China Scholarship Council (201913043).

\section{SUPPLEMENTARY MATERIAL}

The Supplementary Material for this article can be found online at: https://www.frontiersin.org/articles/10.3389/fnut.2021. 719935/full\#supplementary-material

sows. J Anim Sci. (1998) 76:1433-6. doi: 10.2527/1998.7651 $433 \mathrm{x}$

18. Coles LT, Moughan PJ, Awati A, Darragh AJ. Optimisation of inoculum concentration and incubation duration for an in vitro hindgut dry matter digestibility assay. Food Chem. (2013) 136:624-31. doi: 10.1016/j.foodchem.2012.08.045

19. AOAC International. Official Method of Analysis of AOAC International, 18th ed. Gaithersburg, MD: AOAC Int. (2007).

20. Van Soest P, v., Robertson J, Lewis B. Methods for dietary fiber, neutral detergent fiber, and nonstarch polysaccharides in relation to animal nutrition. J Dairy Sci. (1991) 74:3583-97. doi: 10.3168/jds.S0022-0302(91)78551-2

21. Bai Y, Zhao JB, Tao SY, Zhou XJ, Pi Y, Gerrits WJ, et al. Effect of dietary fiber fermentation on short-chain fatty acid production and microbial composition in vitro. J Sci Food Agric. (2020) 100:4282-91. doi: 10.1002/jsfa. 10470

22. Coles LT, Moughan PJ, Awati A, Darragh AJ. Validation of a dual in vivo-in vitro assay for predicting the digestibility of nutrients in humans. J Sci Food Agric. (2013) 93:2637-45. doi: 10.1002/jsf a. 6108

23. Whelan K, Judd PA, Preedy VR, Simmering R, Jann A, Taylor MA. Fructooligosaccharides and fiber partially prevent the alterations in fecal microbiota and short-chain fatty acid concentrations caused by standard enteral formula in healthy humans. J Nutr. (2005) 135:1896-902. doi: $10.1093 /$ jn/135.8.1896

24. Zhao J, Liu X, Ling Z, Junjun W, Zhang S. Effects of body weight and fiber sources on fiber digestibility and short chain fatty acid concentration in growing pigs. Asian-Australas J Anim Sci. (2019) 33:1975-84. doi: 10.5713/ajas.19.0713

25. Topping DL, Clifton PM. Short-chain fatty acids and human colonic function: roles of resistant starch and nonstarch polysaccharides. Physiol Rev. (2001) 81:1031-64. doi: 10.1152/physrev.2001.81.3.1031

26. Christensen DN, Knudsen KEB, Wolstrup J, Jensen BB. Integration of ileum cannulated pigs and in vitro fermentation to quantify the effect of diet composition on the amount of short-chain fatty acids available from fermentation in the large intestine. J Sci Food Agric. (1999) 79:755-62. doi: 10.1002/(SICI)1097-0010(199904)79:5 < 755::AID-JSFA248>3.0.CO;2-2

27. Seema H, Metzler-Zebeli BU, Thavaratnam V, Zijlstra RT. Effects of viscosity and fermentability of dietary fibre on nutrient digestibility and digesta characteristics in ileal-cannulated grower pigs. Br J Nutr. (2011) 106:664-74. doi: 10.1017/S0007114511000985

28. Tannock GW, Liu Y. Guided dietary fibre intake as a means of directing shortchain fatty acid production by the gut microbiota. J Roy Soc New Zeal. (2020) 50:434-55. doi: 10.1080/03036758.2019.1657471

29. Jha R, Bindelle J, Rossnagel B, Kessel AV, Leterme P. In vitro evaluation of the fermentation characteristics of the carbohydrate fractions of hulless barley and other cereals in the gastrointestinal tract of pigs. Anim Feed Sci Tech. (2011) 163:185-93. doi: 10.1016/j.anifeedsci.2010. 10.006

30. Wang JF, Zhu YH, Li DF, Wang Z, Jensen BB. In vitro fermentation of various fiber and starch sources by pig fecal inocula. J Anim Sci. (2004) 82:2615-22. doi: $10.2527 / 2004.8292615 x$

31. Lyu Z, Wang L, Wang J, Wang Z, Zhang S, Wang J, et al. Oat bran and wheat bran impact net energy by shaping microbial communities and fermentation products in pigs fed diets with or without xylanase. J Anim Sci Biotechnol. (2020) 11:99. doi: 10.1186/s40104-020-00505-7

32. Rechkemmer G, Rnnau K, Engelhardt WV. Fermentation of polysaccharides and absorption of short chain fatty acids in the mammalian 
hindgut. Comp Biochem Physiol A Comp Physiol. (1988) 90:563-8. doi: 10.1016/0300-9629(88)90668-8

33. Zhao Y, Tian G, Chen D, Zheng P, Yu J, He J, et al. Dietary protein levels and amino acid supplementation patterns alter the composition and functions of colonic microbiota in pigs. Anim Nutr. (2020) 6:143-51. doi: 10.1016/j.aninu.2020.02.005

Conflict of Interest: YP was employed by company Boen Biotechnology Co. Ltd.

The remaining authors declare that the research was conducted in the absence of any commercial or financial relationships that could be construed as a potential conflict of interest.

The reviewer YZ declared a shared affiliation, with the authors to the handling editor at the time of the review.
Publisher's Note: All claims expressed in this article are solely those of the authors and do not necessarily represent those of their affiliated organizations, or those of the publisher, the editors and the reviewers. Any product that may be evaluated in this article, or claim that may be made by its manufacturer, is not guaranteed or endorsed by the publisher.

Copyright (C) 2022 Bai, Zhou, Zhao, Wang, Ye, Pi, Che, Han, Zhang and Wang. This is an open-access article distributed under the terms of the Creative Commons Attribution License (CC BY). The use, distribution or reproduction in other forums is permitted, provided the original author(s) and the copyright owner(s) are credited and that the original publication in this journal is cited, in accordance with accepted academic practice. No use, distribution or reproduction is permitted which does not comply with these terms. 